\title{
Hierarchies of Stabilizability Preserving Linear Systems
}

\author{
George J. Pappas ${ }^{1}$ \\ Department of Electrical Engineering \\ University of Pennsylvania \\ Philadelphia, PA 19103 \\ pappasg@ee.upenn .edu
}

\author{
Gerardo Lafferriere \\ Department of Mathematical Sciences \\ Portland State University \\ Portland, OR 97207 \\ gerardo@mth.pdx . edu
}

\begin{abstract}
Hierarchical decompositions of control systems are important for reducing the analysis and design of large scale systems. Such decompositions depend on the notion of abstraction: Given a large scale system and a desired property, one tries to extract an abstracted model with equivalent properties, while ignoring details that are irrelevant. Checking the property on the abstraction should be equivalent to checking the property on the original system. In this paper, we focus on large scale linear systems and the property of stabilizability. This results in a hierarchy of linear abstractions that are equivalent from a stabilizability point of view. This is important as high level controller designs are guaranteed to have lower level implementations.
\end{abstract}

\section{Introduction}

Hierarchical control relies on the notions of abstraction or aggregation which refers to grouping the system states into equivalence classes. Depending on the cardinality of the resulting quotient space we may have discrete or continuous abstractions. With this notion of abstraction, the abstracted system can be defined as the induced quotient system. Hierarchical approaches perform analysis or design of the abstracted system, and then refine the design at the lower level while incorporating modeling detail.

Purely discrete abstractions of continuous systems have been considered in $[2,4]$. Hierarchical systems for discrete event systems have been formally considered in [10]. Continuous abstractions of continuous systems is a very recent activity [7]. More precisely, for linear control systems the abstraction problem is formulated as follows.

Problem 1.1 [Linear Abstractions([7])] Given a control system

$$
\dot{x}=A x+B u \quad x \in \mathbb{R}^{n} \quad u \in \mathbb{R}^{m}
$$

\footnotetext{
${ }^{1}$ Research partially supported by DARPA MoBIES Grant F33615-00-C-1707, and by the University of Pennsylvania Research Foundation.
}

and an onto map $y=C x$, define a control system

$$
\dot{y}=F y+G v \quad y \in \mathbb{R}^{p} \quad v \in \mathbb{R}^{k}
$$

which can produce as trajectories all functions of the form $y(t)=C x(t)$, where $x(t)$ is a trajectory of system (1). That is, $C$ maps trajectories of system (1) to trajectories of system (2).

The map $y=C x$ performs the state aggregation. System (2) will be referred to as the abstraction of system (1). Note that the control input $v(t)$ of the coarser model (2) is not the same input $u(t)$ of system (1) and should be thought of as a higher level input. This differentiates abstraction from more traditional model reduction techniques [1] which maintain the same input in the reduction process.

In [7], Problem 1.1 was solved by generalizing the notion of $\Phi$-related vector fields from differential geometry to control systems. Interestingly, Problem 1.1 is always solvable if the matrix $C$ is full row rank. In addition to propagating trajectories from the original system (1) to the abstracted system (2), one is interested in propagating properties from the abstracted system to the original system. This is the complexity reducing direction since checking the property on the simpler system is equivalent to checking the property on the complicated system. More precisely, one is interested in characterizing quotient maps having these desirable properties. In $[6,7]$, we considered various notions of reachability. In this paper, we consider the property of stabilizability.

Problem 1.2 [Stabilizability preserving abstractions] Given the linear control system

$$
\dot{x}=A x+B u \quad x \in \mathbb{R}^{n} \quad u \in \mathbb{R}^{m},
$$

characterize linear maps $y=C x$, so that the abstracted linear system

$$
\dot{y}=F y+G v \quad y \in \mathbb{R}^{p} \quad v \in \mathbb{R}^{k}
$$

is stabilizable if and only if system (3) is stabilizable. 
A solution to the above problem is important for hierarchical stabilization algorithms of large scale linear systems since stabilizability of the original system (3) is guaranteed by stabilizability of the abstracted system (4). Therefore, if such a stabilizability preserving hierarchy is constructed, then one can perform controller synthesis for the abstracted system and refine the controller design at the lower level while accommodating ignored dynamics. This principled way for hierarchical stabilization is clearly related to backstepping designs [5].

This paper is structured as follows: In Section 2 we review some results regarding abstractions of linear systems. They will be used in Section 3 where we develop a solution to Problem 1.2, before offering a variety of issues for further research in Section 4.

\section{Linear Abstractions}

In this section we review all relevant results from [7]. We begin with a formal definition of linear abstractions.

Definition 2.1 [Linear Abstractions([7])] Consider the linear control systems

$$
\begin{array}{llll}
\left(\Sigma_{1}\right) & \dot{x}=A x+B u & x \in \mathbb{R}^{n} \quad u \in \mathbb{R}^{m} \\
\left(\Sigma_{2}\right) & \dot{y}=F y+G v & y \in \mathbb{R}^{p} \quad v \in \mathbb{R}^{k}
\end{array}
$$

and a surjective map $y=C x$. Then control system $\Sigma_{2}$ is called a C-abstraction of system $\Sigma_{1}$ if system $\Sigma_{2}$ can produce as trajectories all functions of the form $y(t)=C x(t)$, where $x(t)$ is a trajectory of system $\Sigma_{1}$.

The definition of linear abstraction relates the trajectories of the two systems. Note that system $\Sigma_{2}$ must capture all (output) trajectories of system $\Sigma_{1}$, but may also generate more trajectories. At the level of vector fields we have the following notion.

Definition 2.2 [C-related linear systems] Consider the linear time-invariant control systems

$$
\begin{array}{lll}
\left(\Sigma_{1}\right) & \dot{x}=A x+B u & x \in \mathbb{R}^{n} \quad u \in \mathbb{R}^{m} \\
\left(\Sigma_{2}\right) & \dot{y}=F y+G v & y \in \mathbb{R}^{p} \quad v \in \mathbb{R}^{k}
\end{array}
$$

and the linear, surjective map $y=C x$. Then $\Sigma_{2}$ is $C$-related to $\Sigma_{1}$ if $\forall x \in \mathbb{R}^{n}, \forall u \in \mathbb{R}^{k}, \exists v \in \mathbb{R}^{l}$ such that

$$
C(A x+B u)=F C x+G v
$$

The connection between $C$-abstractions and $C$-related systems is given by the following theorem which also solves Problem 1.1.

Theorem $2.3[C$-Abstractions and C-related systems ([7])] Consider the linear time-invariant control systems

$$
\begin{array}{llll}
\left(\Sigma_{1}\right) & \dot{x}=A x+B u & x \in \mathbb{R}^{n} \quad u \in \mathbb{R}^{m} \\
\left(\Sigma_{2}\right) & \dot{y}=F y+G v & y \in \mathbb{R}^{p} \quad v \in \mathbb{R}^{k}
\end{array}
$$

and linear, surjective map $y=C x$. Then $\Sigma_{2}$ is a $C$ abstraction of $\Sigma_{1}$ if and only if $\Sigma_{2}$ is C-related to $\Sigma_{1}$.

Given $C$-abstractions and $C$-related systems, it is clearly advantageous to work with $C$-related systems since they potentially offer more constructive ways for generating abstractions. In particular, The following proposition gives us a canonical construction in order to generate $C$-related linear abstractions.

Proposition 2.4 [Canonical construction ([7])] Consider the linear system

$$
\left(\Sigma_{1}\right) \quad \dot{x}=A x+B u
$$

and a surjective map $y=C x$. Let

$$
\left(\Sigma_{2}\right) \quad \dot{y}=F y+G v
$$

be the system where

$$
\begin{aligned}
F & =C A C^{+} \\
G & =\left[\begin{array}{llll}
C B & C A v_{1} & \ldots & C A v_{r}
\end{array}\right]
\end{aligned}
$$

with $C^{+}$the Penrose pseudoinverse of $C$, and $v_{1}, \ldots, v_{r}$ spanning $\operatorname{Ker}(C)$. Then $\Sigma_{2}$ is C-related to $\Sigma_{1}$.

Note that by Proposition 2.4, given any linear control system and full row rank matrix $C$, there always exists another linear control system which is $C$-related to it. In addition to trajectories, we are also interested in propagation of other properties such as controllability. For linear system $\dot{x}=A x+B u$, the reachable space from the origin is given by $\mathcal{R}(A, B)=$ $\operatorname{Im}\left[B A B \ldots A^{n-1} B\right]$. As a corollary of Theorem 2.3 we obtain the following result.

Theorem 2.5 [Controllability Propagation ([7])] Consider the linear systems

$$
\begin{array}{ll}
\left(\Sigma_{1}\right) & \dot{x}=A x+B u \\
\left(\Sigma_{2}\right) & \dot{y}=F y+G v
\end{array}
$$

where $\Sigma_{2}$ is $C$-related to $\Sigma_{1}$ which respect to $y=C x$. Then $C \mathcal{R}(A, B) \subseteq \mathcal{R}(F, G)$. In particular, if $\Sigma_{1}$ is controllable then $\Sigma_{2}$ is controllable.

In order to propagate controllability from the abstracted linear system $\Sigma_{2}$ to the original system $\Sigma_{1}$, conditions must be placed on the abstracting map $y=C x$, resulting in consistent abstractions [7]. With respect to controllability, the following theorem characterizes consistent linear abstractions. 
Theorem 2.6 [Controllability preserving linear abstractions ([7])] Consider the linear system

$$
\left(\Sigma_{1}\right) \quad \dot{x}=A x+B u
$$

and surjective map $y=C x$. Let

$$
\left(\Sigma_{2}\right) \quad \dot{y}=F y+G v
$$

be the $C$-related system where

$$
\begin{aligned}
F & =C A C^{+} \\
G & =\left[\begin{array}{llll}
C B & C A v_{1} & \ldots & C A v_{r}
\end{array}\right]
\end{aligned}
$$

where $C^{+}$is the pseudoinverse of $C$ and $v_{1}, \ldots, v_{r}$ span $\operatorname{Ker}(C)$. Furthermore assume that

$$
\operatorname{Ker}(C) \subseteq \mathcal{R}(A, B)
$$

Then $\Sigma_{1}$ is controllable if and only if $\Sigma_{2}$ is controllable.

The goal of this paper is to examine similar issues and obtain related results for the property of stabilizability. Compared to controllability, stabilizability poses technical challenges as eigenstructure information must also be propagated between the original system and its abstraction, and vice versa.

\section{Stabilizability Preserving Abstractions}

We begin this section by reviewing some standard notions regarding stabilizability. Consider again the linear control system

$$
\left(\Sigma_{1}\right) \quad \dot{x}=A x+B u
$$

where the characteristic polynomial of $A$ is decomposed into a product of polynomials

$$
\operatorname{det}(A-\lambda I)=p_{A}^{-}(\lambda) p_{A}^{+}(\lambda)
$$

where all the roots of $p_{A}^{-}(\lambda)$ have negative real parts, and all the roots of $p_{A}^{+}(\lambda)$ have nonnegative real parts. The stable and unstable subspaces are defined as

$$
\begin{aligned}
& X^{-}=\operatorname{Ker}\left(p_{A}^{-}(A)\right)=\bigoplus_{\left\{\lambda_{k} \mid \operatorname{Re}\left(\lambda_{k}\right)<0\right\}} \operatorname{Ker}\left[\left(A-\lambda_{k} I\right)^{m_{k}}\right] \\
& X^{+}=\operatorname{Ker}\left(p_{A}^{+}(A)\right)=\bigoplus_{\left\{\lambda_{k} \mid \operatorname{Re}\left(\lambda_{k}\right) \geq 0\right\}} \operatorname{Ker}\left[\left(A-\lambda_{k} I\right)^{m_{k}}\right]
\end{aligned}
$$

where $m_{k}$ is the algebraic multiplicity of eigenvalue $\lambda_{k}$. Furthermore, $X^{-}$and $X^{+}$are $A$-invariant subspaces that result in the decomposition $\mathbb{R}^{n}=X^{-} \oplus X^{+}$. The stable subspace and the controllability subspace combine to produce the so called stabilizable subspace

$$
\mathcal{S}(A, B)=X^{-}+\mathcal{R}(A, B)
$$

which is the smallest A-invariant subspace that contains the controllable and stable states. It is well known that system $\Sigma_{1}$ is stabilizable if and only if $\mathcal{S}(A, B)=\mathbb{R}^{n}$. It is useful to think of stabilizability as asymptotic controllability ([3]). The following proposition makes this connection precise.
Proposition 3.1 ([3]) Consider the linear system

$$
\left(\Sigma_{1}\right) \quad \dot{x}=A x+B u
$$

Then $x_{0} \in \mathcal{S}(A, B)$ if and only if there exists a control input $u(t)$ that results in state trajectory $x(t)$ starting from $x_{0}$ such that $\lim _{t \rightarrow+\infty} x(t)=0$.

\subsection{From original to abstracted system}

We now focus on propagating properties from the original system to the abstracted $C$-related system. Given the above characterization of stabilizable subspaces, we can immediately obtain our first result which relates the stabilizable subspaces of $C$-related systems.

Proposition 3.2 [Stabilizability propagation] Consider the linear systems

$$
\begin{array}{ll}
\left(\Sigma_{1}\right) & \dot{x}=A x+B u \\
\left(\Sigma_{2}\right) & \dot{y}=F y+G v
\end{array}
$$

where system $\Sigma_{2}$ is $C$-related to system $\Sigma_{1}$. Then

$$
C \mathcal{S}(A, B) \subseteq \mathcal{S}(F, G)
$$

Therefore, if $\Sigma_{1}$ is stabilizable, then $\Sigma_{2}$ is stabilizable.

Proof: Let $x_{0} \in \mathcal{S}(A, B)$. Then there exists a control input $u(t)$ that results in state trajectory $x(t)$ of $\Sigma_{1}$ from $x_{0}$ such that $\lim _{t \rightarrow+\infty} x(t)=0$. Now consider $y_{0}=C x_{0}$. By the Theorem 2.3, there exists an input $v(t)$ such that the trajectory $y(t)$ of $\Sigma_{2}$ from $y_{0}$ satisfies $y(t)=C x(t)$, and thus $\lim _{t \rightarrow+\infty} y(t)=0=$ $C \lim _{t \rightarrow+\infty} x(t)=0$. Thus $y_{0} \in \mathcal{S}(F, G)$ which concludes the proof.

Since for $C$-related systems we have that $C \mathcal{S}(A, B) \subseteq$ $\mathcal{S}(F, G)$, then in particular $C X^{-} \subseteq Y^{-}+\mathcal{R}(F, G)$. Therefore, for $C$-related systems, the stable subspace of the original system propagates to either the stable subspace or the controllable subspace of the abstracted system. Related to stabilizability, is the concept of controlled invariant subspaces [9].

Definition 3.3 Consider the linear system

$$
\left(\Sigma_{1}\right) \quad \dot{x}=A x+B u \quad x \in \mathbb{R}^{n} \quad u \in \mathbb{R}^{m}
$$

A subspace $\mathcal{V}$ is called controlled invariant or $(A, B)$ invariant if and only if $A \mathcal{V} \subseteq \mathcal{V}+\mathcal{R}(B)$.

The following proposition shows that in $C$-related systems, controlled invariant subspaces propagate to controlled invariant subspaces.

Proposition 3.4 [Propagation of controlled invariant subspaces] Consider the linear systems

$$
\begin{array}{llll}
\left(\Sigma_{1}\right) & \dot{x}=A x+B u & x \in \mathbb{R}^{n} \quad u \in \mathbb{R}^{m} \\
\left(\Sigma_{2}\right) & \dot{y}=F y+G v & y \in \mathbb{R}^{p} \quad v \in \mathbb{R}^{k}
\end{array}
$$


where system $\Sigma_{2}$ is $C$-related to system $\Sigma_{1}$. Let $\mathcal{V}$ be an $(A, B)$-invariant subspace. Then $C \mathcal{V}$ is an $(F, G)$ invariant subspace.

Proof: Recall Definition 2.2 for $C$-related systems. Since for all $x \in \mathbb{R}^{n}, u \in \mathbb{R}^{k}$ there exists $v \in \mathbb{R}^{l}$ such that $C(A x+B u)=F C x+G v$, then for $x=0$ we obtain that $\mathcal{R}(C B) \subseteq \mathcal{R}(G)$. Furthermore for $u=0$, we obtain that for all $x \in \mathbb{R}^{n}$ there exists $v$ such that $F C x=C A x+G v$. Now consider $y=C x$ where $x \in \mathcal{V}$. Then $F y=F C x=C A x+G v$ for some input $v$. By $(A, B)$-invariance of $\mathcal{V}, A x=x^{\prime}+u^{\prime}$ where $x^{\prime} \in \mathcal{V}$ and $u^{\prime} \in \mathcal{R}(B)$. But then $F y=C x^{\prime}+C u^{\prime}+G v$ where $C x^{\prime} \in$ $C \mathcal{V}$, and $C u^{\prime} \in \mathcal{R}(C B) \subseteq \mathcal{R}(G)$ and $G v \in \mathcal{R}(G)$. This completes the proof.

In general, it is not true that for $C$-related systems, $A$-invariant subspaces propagate to $F$-invariant subspaces. However, if our $C$-related systems are constructed using the canonical approach of Proposition 2.4, then invariant subspaces propagate in a particular way. Since $F=C A C^{+}$, we have that $F C x=C A x$ for all $x \in \operatorname{Ker}(C)^{\perp}$.

Lemma 3.5 Let $F=C A C^{+}$where $C$ is full row rank, $\mathcal{V} \subseteq \mathbb{R}^{n}$ be any subspace, and define the subspace $\mathcal{W}=$ $C \mathcal{V}$. Then

$$
A \mathcal{V} \subseteq \mathcal{V} \Longrightarrow F \mathcal{W} \subseteq \mathcal{W}+C A K \operatorname{er}(C)
$$

Therefore, if $A \operatorname{Ker}(C) \subseteq \operatorname{Ker}(C)+\mathcal{V}$ then $F \mathcal{W} \subseteq \mathcal{W}$.

Proof: Let $y \in \mathcal{W}$, that is $y=C x$ where $x \in \mathcal{V}$. Then $F C x=F C\left(x_{c}+x_{n}\right)$ where $x_{c} \in \operatorname{Ker}(C)$ and $x_{n} \in \operatorname{Ker}(C)^{\perp}$. Thus $F C x=F C x_{n}$. By the comment above $F C x=F C x_{n}=C A x_{n}=C A\left(x-x_{c}\right)=C A x-$ $C A x_{c}$. By assumption, $A x \in \mathcal{V}$. Therefore $F y \in \mathcal{W}+$ $C A \operatorname{Ker}(C)$. Furthermore, if $A \operatorname{Ker}(C) \subseteq \operatorname{Ker}(C)+\mathcal{V}$ then $\mathcal{W}+C A K \operatorname{Ker}(C) \subseteq \mathcal{W}+C \operatorname{Ker}(C)+C \mathcal{V} \subseteq \mathcal{W}$.

Conversely we also have the following.

Lemma 3.6 Let $F=C A C^{+}$where $C$ is onto, $\mathcal{W} \subseteq$ $\mathbb{R}^{p}$ be any subspace, and define the subspace $\mathcal{V}=$ $C^{-1}(\mathcal{W}) \subseteq \mathbb{R}^{n}$. Then

$$
F \mathcal{W} \subseteq \mathcal{W} \Longrightarrow A \mathcal{V} \subseteq \mathcal{V}+A K \operatorname{Ker}(C)
$$

In particular, if $A \operatorname{Ker}(C) \subseteq \mathcal{V}$ then $A \mathcal{V} \subseteq \mathcal{V}$

Proof: $\quad$ Let $x \in \mathcal{V}=C^{-1}(\mathcal{W})$, that is $y=C x \in \mathcal{W}$. Then $x=C^{+} y+x_{c}$ where $x_{c} \in \operatorname{Ker}(C)$. But then $A x=A\left(C^{+} y+x_{c}\right)=A C^{+} y+A x_{c}$. But $A C^{+} y \in$ $\mathcal{V}=C^{-1}(\mathcal{W})$ since $C A C^{+} y=F y \in \mathcal{W}$ by assumption. Thus $A x=A C^{+} y+A x_{c} \in \mathcal{V}+A K \operatorname{Ker}(C)$.

\subsection{From abstracted to original system}

At this point, we would like to start propagating properties related to stabilizability from the abstracted system to the original system. We begin by the notion of implementability.

Definition 3.7 [Stabilizability Implementation] Consider the linear time-invariant control systems

$$
\begin{array}{lll}
\left(\Sigma_{1}\right) & \dot{x}=A x+B u & x \in \mathbb{R}^{n} \quad u \in \mathbb{R}^{m} \\
\left(\Sigma_{2}\right) & \dot{y}=F y+G v & y \in \mathbb{R}^{p} \quad v \in \mathbb{R}^{k}
\end{array}
$$

where $\Sigma_{2}$ is C-related to $\Sigma_{1}$ which respect to $y=C x$. Then $\Sigma_{1}$ is an implementation of $\Sigma_{2}$ if the following property holds: whenever there exists a trajectory $y(t)$ of $\Sigma_{2}$ starting at some $y_{0}$ with $\lim _{t \longrightarrow+\infty} y(t)=0$, then there exists some $x_{0} \in C^{-1}\left(y_{0}\right)$ and a $\Sigma_{1}$ trajectory $x(t)$ starting from $x_{0}$ with $\lim _{t \rightarrow+\infty} x(t)=0$.

Notice that implementability is an existential property, and asks the lower level system to reach the origin for some $x_{0} \in C^{-1}\left(y_{0}\right)$ (but not for all such $x_{0}$ ). In order for the property of reaching asymptotically the origin to be independent of the particular choice of $x_{0} \in C^{-1}\left(y_{0}\right)$, we define the notion of consistency.

Definition 3.8 [Stabilizability Consistency] The linear control system

$$
\left(\Sigma_{1}\right) \quad \dot{x}=A x+B u \quad x \in \mathbb{R}^{n} \quad u \in \mathbb{R}^{m}
$$

is consistent with respect to $y=C x$ if the following holds: if there is a trajectory of $\Sigma_{1}$ asymptotically connecting $x_{1}$ to the origin, then for any $x_{2}$ with $C x_{1}=C x_{2}$ there exists a trajectory of $\Sigma_{1}$ that asymptotically connects $x_{2}$ to the origin.

Consistency simply says that our ability to asymptotically reach the origin is independent of the choice of $x \in C^{-1}(y)$. The notions of implementability and consistency can be merged in a straightforward manner in order to propagate stabilizability from the abstracted to the original system.

Theorem 3.9 [Implementability +Consistency] Consider the linear control systems

$$
\begin{array}{lll}
\left(\Sigma_{1}\right) & \dot{x}=A x+B u & x \in \mathbb{R}^{n} \quad u \in \mathbb{R}^{m} \\
\left(\Sigma_{2}\right) & \dot{y}=F y+G v & y \in \mathbb{R}^{p} \quad v \in \mathbb{R}^{k}
\end{array}
$$

where $\Sigma_{2}$ is $C$-related to $\Sigma_{1}$. Furthermore, assume that $\Sigma_{1}$ implements $\Sigma_{2}$, and $\Sigma_{1}$ is consistent. Then $\Sigma_{1}$ is stabilizable if and only if $\Sigma_{2}$ is stabilizable.

Proof: We already know from Proposition 3.2 that if $\Sigma_{1}$ is stabilizable then $\Sigma_{2}$ is stabilizable. Now consider any $x_{0} \in \mathbb{R}^{n}$ and let $y_{0}=C x_{0}$. By assumption, $\Sigma_{2}$ is stabilizable, so there exists control input $v(t)$ and a 
$\Sigma_{2}$ trajectory $y(t)$ which asymptotically converges to the origin. Since $\Sigma_{1}$ implements $\Sigma_{2}$, there exists some $x_{1} \in C^{-1}\left(y_{0}\right)$ and a trajectory $x(t)$ of $\Sigma_{1}$ that results in $\lim _{t \rightarrow+\infty} x(t)=0$. But then $y_{0}=C x_{0}=C x_{1}$ and by consistency of $\Sigma_{1}$ there must also exist a trajectory of $\Sigma_{1}$ that results in $x_{0}$ reaching asymptotically the origin. Thus $\Sigma_{1}$ is stabilizable.

We now obtain concrete algebraic characterization of implementability. The proof is a direct consequence of Theorem 3.2, Definition 3.7, and Proposition 3.1.

Proposition 3.10 [Implementation Characterization] Consider the linear control systems

$$
\begin{array}{lll}
\left(\Sigma_{1}\right) & \dot{x}=A x+B u & x \in \mathbb{R}^{n} \quad u \in \mathbb{R}^{m} \\
\left(\Sigma_{2}\right) & \dot{y}=F y+G v & y \in \mathbb{R}^{p} \quad v \in \mathbb{R}^{k}
\end{array}
$$

where $\Sigma_{2}$ is C-related to $\Sigma_{1}$. Then $\Sigma_{1}$ is an implementation of $\Sigma_{2}$ if and only if

$$
C \mathcal{S}(A, B)=\mathcal{S}(F, G)
$$

Proposition 3.11 [Consistency Consider the linear control system

$$
\left(\Sigma_{1}\right) \quad \dot{x}=A x+B u \quad x \in \mathbb{R}^{n} \quad u \in \mathbb{R}^{m}
$$

Then $\Sigma_{1}$ is consistent with respect to $y=C x$ iff

$$
\operatorname{Ker}(C) \subseteq \mathcal{S}(A, B)
$$

Proof: Definition 3.8 requires that if $x_{0} \in \mathcal{S}(A, B)$ then $x_{0}+\operatorname{Ker}(C) \in \mathcal{S}(A, B)$ therefore a characterization of consistency for stabilizability is simply $\operatorname{Ker}(C) \subseteq \mathcal{S}(A, B)$.

Theorem 3.9 requires that $\Sigma_{1}$ implements $\Sigma_{2}$, and that $\Sigma_{2}$ is consistent. Satisfying both characterizations of Propositions 3.10 and 3.11 results in one condition.

Theorem 3.12 Consider the linear control system

$$
\left(\Sigma_{1}\right) \quad \dot{x}=A x+B u
$$

surjective map $y=C x$, and let

$$
\left(\Sigma_{2}\right) \quad \dot{y}=F y+G v
$$

be C-related. Then $\Sigma_{1}$ implements $\Sigma_{2}$, and $\Sigma_{1}$ is consistent if and only if

$$
\mathcal{S}(A, B)=C^{-1}(\mathcal{S}(F, G))
$$

For general $C$-related systems, if condition (8) is satisfied, then $\Sigma_{1}$ is stabilizable if and only if $\Sigma_{2}$ is stabilizable. Checking condition (8) may be difficult. Our eventual goal is to simply have check the consistency condition (7) for the canonical construction of Proposition 2.4. To achieve this, we first show that for general $C$-related systems, the following weaker condition is sufficient for propagating stabilizability from the abstracted to the original system.
Theorem 3.13 Consider the linear control system

$$
\left(\Sigma_{1}\right) \quad \dot{x}=A x+B u
$$

surjective map $y=C x$, and let

$$
\left(\Sigma_{2}\right) \quad \dot{y}=F y+G v
$$

be C-related to $\Sigma_{1}$. If

$$
C^{-1}(\mathcal{R}(F, G)) \subseteq \mathcal{S}(A, B)
$$

then $\Sigma_{2}$ is stabilizable if and only if $\Sigma_{1}$ is stabilizable.

Proof: One direction is given to us by Proposition 3.2. Now decompose $\mathbb{R}^{n}=\mathcal{R}(A, B) \oplus$ $\mathcal{R}(A, B)^{\perp}$ and $\mathbb{R}^{m}=\mathcal{R}(F, G) \oplus \mathcal{R}(F, G)^{\perp}$ using the basis induced by the respective Kalman decompositions. In these bases the matrices take the form $A=\left(\begin{array}{cc}A_{11} & A_{12} \\ 0 & A_{22}\end{array}\right), \quad F=\left(\begin{array}{cc}F_{11} & F_{12} \\ 0 & F_{22}\end{array}\right), \quad B=$ $\left(\begin{array}{c}B_{1} \\ 0\end{array}\right), \quad G=\left(\begin{array}{c}G_{1} \\ 0\end{array}\right), \quad C=\left(\begin{array}{ll}C_{11} & C_{12} \\ C_{21} & C_{22}\end{array}\right)$ with the appropriate dimensions. By Theorem 2.5, we have $C \mathcal{R}(A, B) \subseteq \mathcal{R}(F, G)$. By the special form of our matrices this implies that $C_{21} \mathcal{R}\left(A_{11}, B_{1}\right)=0$. Since by construction $\mathcal{R}\left(A_{11}, B_{1}\right)$ is full (row) rank we conclude that $C_{21}=0$.

By Definition 2.2, for all $x \in \mathbb{R}^{n}$ we have that $(F C-$ $C A) x \in \mathcal{R}(G)$. Since $C_{21}=0$, the structure of the matrices above results in $F_{22} C_{22}=C_{22} A_{22}$. Assume that $\Sigma_{2}$ is stabilizable, or otherwise that $F_{22}$ is Hurwitz. Our goal is to show that $A_{22}$ is Hurwitz. Let $\lambda$ be an eigenvalue of $A_{22}$ with corresponding eigenvector $x_{2} \neq 0$. Then $F_{22} C_{22} x_{2}=C_{22} A_{22} x_{2}=\lambda C_{22} x_{2}$. If $C_{22} x_{2} \neq 0$ then it is an eigenvector for $F_{22}$ and so $\operatorname{Re}[\lambda]<0$. If $C_{22} x_{2}=0$ then the vector $x=\left(\begin{array}{c}0 \\ x_{2}\end{array}\right)$ satisfies $C x \in \mathcal{R}(F, G)$, since the last coordinates are zero. Then by assumption $x \in \mathcal{S}(A, B)$ and so we can write $x=r_{c}+r_{s}$ where $r_{c} \in \mathcal{R}(A, B)$ and $r_{s} \in X^{-}$. But then $r_{c}=\left(\begin{array}{c}r_{1} \\ 0\end{array}\right)$ and so $r_{s}=\left(\begin{array}{l}x_{1} \\ x_{2}\end{array}\right)$. By definition of $X^{-}$we get $p_{A}^{-}(A)\left(\begin{array}{l}x_{1} \\ x_{2}\end{array}\right)=0$. Given the structure of our matrices this means $p_{A}^{-}(A)\left(\begin{array}{l}x_{1} \\ x_{2}\end{array}\right)=$ $\left(\begin{array}{cc}p_{A}^{-}\left(A_{11}\right) & * \\ 0 & p_{A}^{-}\left(A_{22}\right)\end{array}\right)\left(\begin{array}{l}x_{1} \\ x_{2}\end{array}\right)=\left(\begin{array}{c}* \\ p_{A}^{-}\left(A_{22}\right) x_{2}\end{array}\right)=0$. Therefore $p_{A}^{-}\left(A_{22}\right) x_{2}=0$. Since $A_{22} x_{2}=\lambda x_{2}$ we get $p_{A}^{-}\left(A_{22}\right) x_{2}=p_{A}^{-}(\lambda) x_{2}=0$ which in turn implies $p_{A}^{-}(\lambda)=0$. So $\operatorname{Re}[\lambda]<0$ as desired.

Condition (9) may be difficult to check. Fortunately, for the canonical construction of Proposition 2.4, the consistency condition implies condition (9).

Theorem 3.14 [Consistency implies Stabilizability Equivalence] Consider the C-related linear systems

$$
\left(\Sigma_{1}\right) \quad \dot{x}=A x+B u
$$




$$
\left(\Sigma_{2}\right) \quad \dot{y}=F y+G v
$$

where $\Sigma_{2}$ is obtained using the canonical construction

$$
\begin{aligned}
F & =C A C^{+} \\
G & =\left[\begin{array}{llll}
C B & C A v_{1} & \ldots & C A v_{r}
\end{array}\right]
\end{aligned}
$$

where $C^{+}$is the Moore-Penrose pseudoinverse of $C$, and $v_{1}, \ldots, v_{r}$ span $\operatorname{Ker}(C)$. Furthermore assume that

$$
\operatorname{Ker}(C) \subseteq S(A, B)
$$

Then $\Sigma_{1}$ is stabilizable if and only if $\Sigma_{2}$ is stabilizable.

Proof: For the canonical construction, Lemma 3.5 results in $C \mathcal{S}(A, B)$ being $F$-invariant. We now show that $\mathcal{R}(F, G) \subseteq C \mathcal{S}(A, B)$. Since for the canonical construction we have $G=\left[\begin{array}{ll}C B & C A K \operatorname{Ker}(C)\end{array}\right]$, and by assumption $\operatorname{Ker}(C) \subseteq \mathcal{S}(A, B)$, then we get that $\mathcal{R}(G)=C(\mathcal{R}(B)+A \bar{K} e r(C)) \subseteq C(\mathcal{R}(B)+$ $\mathcal{S}(A, B)) \subseteq C \mathcal{S}(A, B)$. Since $\mathcal{R}(G) \subseteq C \mathcal{S}(A, B)$ and $C \mathcal{S}(A, B)$ is $F$-invariant, then $\mathcal{R}(F G) \subseteq$ $C \mathcal{S}(A, B), \ldots \mathcal{R}\left(F^{m-1} G\right) \subseteq C \mathcal{S}(A, B)$, and therefore $\mathcal{R}(F, G) \subseteq C \mathcal{S}(A, B)$. But then $C^{-1}(\mathcal{R}(F, G)) \subseteq$ $C^{-1} C \mathcal{S}(A, B) \subseteq \mathcal{S}(A, B)+\operatorname{Ker}(C) \subseteq \mathcal{S}(A, B)$. Thus condition (9) is satisfied, and Theorem 3.13 applies.

In other words, the consistency condition of Theorem 3.14 , states that in order to preserve stabilizability, then the directions that we must ignore $(\operatorname{Ker}(C))$, must be either stable $\left(X^{-}\right)$or controllable $(\mathcal{R}(A, B))$. Furthermore, we can always perform such consistent abstractions as long as control inputs exist.

If $\operatorname{Ker}(C)$ is $A$-invariant, then we can use a stabilizing feedback for $F$ to partially stabilize $A$. To illustrate this assume that $(A, B)$ is controllable. Then the $C$-related system $(F, G)$ is also controllable. Let $L$ be a matrix such that $F+G L$ is Hurwitz and define $K=L C$. (Remember that in this case $G=C B$.) Notice first that $\operatorname{Ker}(C)$ is $(A+B K)$-invariant and that $C(A+B K) C^{+}=F+B L$. So $F+G L$ is the induced canonical abstraction of $A+B K$ (the quotient vector field) and they are hence $C$-related. A direct calculation shows that the spectrum of $A+B K$ is the union of the spectrum of $F+G L$ and that of the restriction of $A+B K$ to $\operatorname{Ker}(C)$ (see also the proof of Theorem 3.13). If, in addition, $\operatorname{Ker}(C)^{\perp}$ is $A$-invariant then this choice of $K$ stabilizes the restriction of $A$ to $\operatorname{Ker}(C)^{\perp}$. Let $P$ be an orthogonal matrix whose columns span $\operatorname{Ker}(C)$. If the pair $\left(P^{T} A P, P^{T} B\right)$ is also controllable then the modes in $\operatorname{Ker}(C)$ can also be stabilized resulting in an effective hierarchical stabilization procedure. The approach would start with an $A$-invariant subspace $\mathcal{V}$, choose $C$ with $\operatorname{Ker}(C)=\mathcal{V}$ and then use the canonical construction for the $C$-related system $(F, G)$. The numerically stable procedure of [8] for partial pole placement essentially corresponds to defining $C=Q^{T}$ where the columns of $Q$ form an ortonormal basis of $\mathcal{V}^{\perp}$.

\section{Conclusions}

In this paper, we considered the problem of stabilizability preserving abstractions for linear systems, and characterized stabilizability preserving aggregation maps. These results inspire a hierarchical stabilizability algorithm, as well as hierarchical controller design algorithms. To achieve this we need to better understand how feedback gains at the abstracted level can be refined to the original system. The nonlinear analogues of the results of this paper are of clear relevance and importance to backstepping designs.

\section{References}

[1] M. Aoki. Control of large scale dynamic systems by aggregation. IEEE Transactions on Automatic Control, 13(3):246-253, June 1968.

[2] P.E. Caines and Y.J. Wei. Hierarchical hybrid control systems: A lattice theoretic formulation. IEEE Transactions on Automatic Control : Special Issue on Hybrid Systems, 43(4):501-508, April 1998.

[3] F. M. Callier and C. Desoer. Linear System Theory. Springer-Verlag, 1991.

[4] J.E.R. Cury, B.H. Krogh, and T. Niinomi. Synthesis of supervisory controllers for hybrid systems based on approximating automata. IEEE Transactions on Automatic Control : Special Issue on Hybrid Systems, 43(4):564-568, April 1998.

[5] M. Kristic, I. Kanellakopoulos, and P. Kokotovic. Nonlinear and Adaptive Control Design. Adaptive and Learning systems for signal processing, communications and control. John Wiley \& Sons, New York, 1995.

[6] G. J. Pappas and S. Simic. Consistent hierarchies of nonlinear abstractions. In Proceedings of the 39th IEEE Conference in Decision and Control, pages 43794384, Sydney, Australia, December 2000.

[7] G.J. Pappas, G. Lafferriere, and S. Sastry. Hierarchically consistent control systems. IEEE Transactions on Automatic Control, 45(6):1141-1160, June 2000.

[8] Y. Saad. Projection and deflation methods for partial pole assignment in linear state feedback. IEEE Transactions on Automatic Control, 33(3):290-297, March 1988.

[9] W.M. Wonham. Linear Multivariable Control : A Geometric Approach, volume 10 of Applications of Mathematics. Springer-Verlag, New York, 1985.

[10] H. Zhong and W.M. Wonham. On the consistency of hierarchical supervision in discrete-event systems. IEEE Transactions on Automatic Control, 35(10):1125-1134, 1990. 